\title{
Arterial spin labelling MRI for assessment of cerebral perfusion in children with moyamoya disease: comparison with dynamic susceptibility contrast MRI
}

\author{
Goetti, Robert ; O’Gorman, Ruth ; Khan, Nadia ; Kellenberger, Christian J ; Scheer, Ianina
}

\begin{abstract}
INTRODUCTION: This study seeks to evaluate the diagnostic accuracy of cerebral perfusion imaging with arterial spin labelling (ASL) MR imaging in children with moyamoya disease compared to dynamic susceptibility contrast (DSC) imaging. METHODS: Ten children (7 females; age, $9.2 \pm 5.4$ years) with moyamoya disease underwent cerebral perfusion imaging with ASL and DSC on a 3-T MRI scanner in the same session. Cerebral perfusion images were acquired with ASL (pulsed continuous 3D ASL sequence, 32 axial slices, $\mathrm{TR}=5.5 \mathrm{~s}, \mathrm{TE}=25 \mathrm{~ms}, \mathrm{FOV}=24 \mathrm{~cm}$, matrix $=128 \times 128)$ and $\mathrm{DSC}$ (gradient echo EPI sequence, 35 volumes of 28 axial slices, TR $=2,000 \mathrm{~ms}$, TE $=36 \mathrm{~ms}, \mathrm{FOV}=24 \mathrm{~cm}$, matrix $=96 \times 96,0.2 \mathrm{ml} / \mathrm{kg}$ Gd-DOTA). Cerebral blood flow maps were generated. ASL and DSC images were qualitatively assessed regarding perfusion of left and right ACA, MCA, and PCA territories by two independent readers using a 3-point-Likert scale and quantitative relative cerebral blood flow (rCBF) was calculated. Correlation between ASL and DSC for qualitative and quantitative assessment and the accuracy of ASL for the detection of reduced perfusion per territory with DSC serving as the standard of reference were calculated. RESULTS: With a good interreader agreement $(=0.62)$ qualitative perfusion assessment with ASL and DSC showed a strong and significant correlation $(=0.77$; $p<0.001)$, as did quantitative $\mathrm{rCBF}(\mathrm{r}=0.79 ; \mathrm{p}<0.001)$. ASL showed a sensitivity, specificity and accuracy of $94 \%$, $93 \%$, and $93 \%$ for the detection of reduced perfusion per territory. CONCLUSION: In children with moyamoya disease, unenhanced ASL enables the detection of reduced perfusion per vascular territory with a good accuracy compared to contrast-enhanced DSC.
\end{abstract}

DOI: https://doi.org/10.1007/s00234-013-1155-8

Posted at the Zurich Open Repository and Archive, University of Zurich

ZORA URL: https://doi.org/10.5167/uzh-77229

Journal Article

Accepted Version

Originally published at:

Goetti, Robert; O'Gorman, Ruth; Khan, Nadia; Kellenberger, Christian J; Scheer, Ianina (2013). Arterial spin labelling MRI for assessment of cerebral perfusion in children with moyamoya disease: comparison with dynamic susceptibility contrast MRI. Neuroradiology, 55(5):639-647.

DOI: https://doi.org/10.1007/s00234-013-1155-8 
Arterial Spin Labeling MRI for Assessment of Cerebral Perfusion in

Children with Moyamoya Disease: Comparison with Dynamic Susceptibility Contrast MRI 


\section{Abstract}

Purpose: To evaluate the diagnostic accuracy of cerebral perfusion imaging with arterial spin labeling (ASL) MR imaging in children with moyamoya disease compared to dynamic susceptibility contrast (DSC) imaging.

Methods: 10 children (7 females, age: 9.2 \pm 5.4 years) with moyamoya disease underwent cerebral perfusion imaging with ASL and DSC on a 3T MRI scanner in the same session. Cerebral perfusion images were acquired with ASL (pulsed continuous 3D ASL sequence, 32 axial slices, $\mathrm{TR}=5.5 \mathrm{~s}, \mathrm{TE}=25 \mathrm{~ms}, \mathrm{FOV}=24 \mathrm{~cm}$, matrix $=128 \mathrm{x} 128$ ) and DSC (gradient echo EPI sequence, 35 volumes of 28 axial slices, $\mathrm{TR}=2000 \mathrm{~ms}, \mathrm{TE}=36 \mathrm{~ms}, \mathrm{FOV}=24 \mathrm{~cm}$, matrix $=96 \times 96$, $0.2 \mathrm{ml} / \mathrm{kg}$ Gd-DOTA). Cerebral blood flow maps were generated. ASL and DSC images were qualitatively assessed regarding perfusion of left and right ACA, MCA and PCA territories by two independent readers using a 3-point-Likert scale and quantitative relative cerebral blood flow (rCBF) was calculated. Correlation between ASL and DSC for qualitative and quantitative assessment and the accuracy of ASL for the detection of reduced perfusion per territory with DSC serving as the standard of reference were calculated.

Results: With a good interreader agreement $(\mathrm{k}=0.62)$ qualitative perfusion assessment with ASL and DSC showed a strong and significant correlation $(\rho=0.77 ; p<0.001)$, as did quantitative $\mathrm{rCBF}(\mathrm{r}=0.79 ; \mathrm{p}<0.001)$. ASL showed a sensitivity, specificity and accuracy of $94 \%, 93 \%$ and $93 \%$ for the detection of reduced perfusion per territory.

Conclusion: In children with moyamoya disease, unenhanced ASL enables the detection of reduced perfusion per vascular territory with a good accuracy compared to contrast-enhanced DSC.

\section{Key words:}

arterial spin labeling; dynamic susceptibility contrast; MRI; moyamoya; pediatric 


\section{Introduction}

Moyamoya disease is a progressive steno-occlusive disease of the the supraclinoid internal cerebral arteries (ICA) and circle of Willis with bilateral involvement of the anterior cerebral arteries (ACA), middle cerebral arteries (MCA) and - to a much lesser degree - the posterior cerebral arteries (PCA), with formation of typical collateral vessel networks, termed "moyamoya" vessels [1] (Fig. 1). The disease generally presents in childhood, with headaches, transient ischemic attacks, strokes and/or epileptic seizures [2]. The treatment of choice consists of surgical revascularization by means of either direct bypass surgery from the superficial temporal artery (STA) to distal branches of the ACA or MCA, or in cases where the donor/recipient vessels are too small in caliber, indirect revascularization procedures such as encephaloduroarteriosynangiosis (EDAS) or encephalogaleaperiostsynangiosis (EGPS) [3-

6]. For both pre- and postoperative assessment of children with moyamoya disease, a noninvasive, non-ionizing imaging procedure providing cerebral perfusion information is of utmost interest.

Dynamic susceptibility contrast imaging (DSC) is the currently most widely used MRI technique for cerebral perfusion imaging and is based on the measurement of the decrease of T2/T2* times of cerebral tissue during the first pass of gadolinium-based contrast agents through the capillary bed [7]. In patients with moyamoya disease, it has been shown to correlate with findings from invasive six-vessel cerebral angiography, nuclear medicine perfusion studies and clinical outcome after revascularization [8; 9]. 
Arterial spin labeling (ASL) is a completely non-invasive MRI technique for cerebral perfusion imaging, which is based on the T1 magnetization state of freely diffusible spinlabelled arterial water. The longitudinal magnetization of water in arterial blood is electromagnetically tagged with radiofrequency pulses and after a predefined transit time, the amount of labelled blood arriving in the tissue of interest is calculated by subtraction from a previously acquired control image [7]. Cerebral blood flow measurements using ASL have been shown to correlate with those of single-photon emission CT (SPECT) in patients with moyamoya disease [10] and with positron emission tomography (PET) in patients with symptomatic carotid artery occlusion [11].

Besides not depending on an exogenous tracer and therefore being completely noninvasive as well as repeatable, ASL offers the possibility of absolute quantification of cerebral blood flow (CBF), whereas absolute quantification with DSC is quite challenging mainly due to non-linear contrast relaxivity [12] and uncertainties regarding the arterial input function [13]. Therefore, with DSC measurements of relative cerebral blood flow (rCBF) rather than absolute cerebral blood flow are generally performed. The possibility of absolute quantification of CBF by ASL may be of use in serial follow-up of children with moyamoya disease. However, the comparability of cerebral perfusion imaging by ASL and the more widely applied and validated DSC has not yet been assessed in children with moyamoya disease. The purpose of our study was therefore to evaluate the correlation of rCBF using ASL and DSC and to assess the diagnostic accuracy of ASL for the detection of reduced perfusion per territory with DSC serving as the standard of reference. 


\section{Materials and Methods}

\section{Patients}

Ten consecutive children ( 7 females, 3 males, age: $9.2 \pm 5.4$ years, range: $1-16$ years) with angiographically proven moyamoya disease who underwent MRI for structural and cerebral perfusion assessment at our institution from August 2011 to July 2012 were included in this retrospective study. Inclusion criteria were angiographically proven moyamoya disease and patient age $\leq 16$ years. Exclusion criteria were postoperative status $<6$ months ( $\mathrm{n}=0$ ), contraindications to sedation in children $<6$ years old as determined by a board-certified anesthesiologist $(\mathrm{n}=0)$, contraindications to contrast-enhanced MRI, such as non-compatible implants $(n=0)$ or known previous allergic reactions to gadolinium-based contrast material $(n=0)$ and non-completion of the imaging protocol $(n=0)$. The indications for MRI were initial assessment prior to possible revascularization measures $(n=5)$ and postoperative follow-up after revascularization $(n=5)$. In the postoperative patients the mean time interval since the operation was $3.6 \pm 4.2$ years (range: 6 months -10 years). They had undergone either bilateral STA-MCA bypass, bilateral EDAS and bilateral EGPS $(\mathrm{n}=1)$, bilateral STA-MCA bypass and bilateral EDAS $(n=1)$, bilateral STA-MCA bypass alone $(n=2)$ or unilateral leftsided STA-MCA bypass, EDAS and EGPS ( $\mathrm{n}=1)$. Institutional review board approval was obtained and the informed consent requirement was waived due to the retrospective nature of the study.

\section{Imaging protocol}

All patients underwent MRI on a 3 Tesla clinical scanner (HD.xt TwinSpeed, GE Medical Systems, Milwaukee, WI, USA) using an 8-channel receive-only head coil for signal reception. For patients under the age of 6 years $(n=4)$, the scan was performed in sedation 
with diisopropylphenol (Propofol, Braun Medical, Melsungen, Germany) closely monitored by a board-certified anesthesiologist throughout the examination.

The scans consisted of our institution's standard protocol for patients with moyamoya disease including triplanar T2-weighted, axial T1-weighted, coronal fluid-attenuated inversion recovery, axial 3D time-of-flight angiography, axial diffusion-weighted imaging, ASL, DSC and post-contrast triplanar T1-weighted imaging. As for the present study only ASL and DSC images were assessed, the remaining sequences of the standard protocol are not further discussed.

ASL was performed with a background-suppressed, pulsed continuous arterial spin labeling (pCASL) sequence, using a 3D stack of spirals fast spin echo readout [14]. 32 axial slices were collected with $\mathrm{TR}=5500 \mathrm{~ms}, \mathrm{TE}=25 \mathrm{~ms}$, slice thickness $=5 \mathrm{~mm}, \mathrm{FOV}=24 \mathrm{~cm}$, and matrix $=128 \times 128$. The labeling duration was $1500 \mathrm{~ms}$ and a post-labeling delay of 1500 ms was used to reduce errors from transit time effects [15]. Vascular crushing gradients were not applied.

DSC was performed using a gradient echo EPI sequence. 35 volumes of 28 axial slices were collected with $\mathrm{TR}=2000 \mathrm{~ms}, \mathrm{TE}=36 \mathrm{~ms}$, slice thickness $=5 \mathrm{~mm}, \mathrm{FOV}=24 \mathrm{~cm}$ and matrix $=96 \times 96$ during manual bolus-injection of $0.2 \mathrm{ml} / \mathrm{kg}$ Gd-DOTA (Dotarem, Guerbet, Roissy, France) in to an antecubital vein at a rate of $2 \mathrm{ml} / \mathrm{s}$, followed by a saline flush.

All patients underwent MRI imaging without complications and yielding diagnostic image quality in all sequences. 


\section{Image analysis}

Images were analysed on a commercially available dedicated workstation (Advantage Workstation, Software version 4.3, GE Medical Systems, Milwaukee, WI, USA). Cerebral blood flow maps were generated from both ASL and DSC data. For ASL, quantitative CBF maps were calculated using the method proposed by Wang et al. [16] with an additional factor included to correct for incomplete recovery of the magnetization in the reference image due to a saturation pulse applied two seconds before imaging [17]. The perfusion was calculated according to the following equation [15] [17].

$$
f=\frac{\lambda}{2 \alpha T_{1 \mathrm{~b}}\left(1-e^{-\frac{\tau}{T_{\mathrm{bb}}}}\right)} \frac{\left(S_{\mathrm{ctrl}}-S_{\mathrm{lbl}}\right)\left(1-e^{-\frac{t_{\mathrm{sat}}}{T_{\mathrm{lg}}}}\right)}{S_{\mathrm{ref}}} e^{\frac{w}{T_{\mathrm{lb}}}}
$$

, where $\mathrm{f}$ is the perfusion (in $\mathrm{ml} / \mathrm{min} / 100 \mathrm{ml}$ ), Sctrl-Slbl is the difference image (control-label), and Sref is a proton-density weighted reference image. $\lambda$ is the blood brain partition coefficient (0.9), $\alpha$ is the inversion efficiency, T1b is the T1 of blood (1600 ms), T1 $\mathrm{g}$ is the $\mathrm{T} 1$ of grey matter $(1200 \mathrm{~ms}), \mathrm{w}$ is the post-labelling delay (1.5 seconds), and $\tau$ is the labelling duration $(1.5 \mathrm{~s})$. The labelling efficiency is given by the product of the PCASL labelling efficiency (0.95) and an additional efficiency factor which incorporates the loss of efficiency from the background suppression (0.75). This model assumes that the labelled spins remain primarily in the microvasculature rather than exchanging with tissue water, so the $\mathrm{T} 1$ of blood is used for quantification [16] [17].

For DSC, rCBF maps were automatically calculated based on a gamma variate fit of the timeconcentration curve using the commercially available scanner software (Functool BrainStat, Software version 4.3, GE Medical Systems, Milwaukee, WI, USA). 


\section{Qualitative image analysis}

ASL and DSC cerebral blood flow maps were independently evaluated by two independent and blinded radiologists with 4 years and 20 years of experience, respectively, regarding image quality (diagnostic vs. non-diagnostic, i.e. due to technical issues or motion artifacts) and cerebral perfusion was visually assessed in bilateral ACA, MCA and PCA territories at three different levels (level of the tentorium, level of the thalami and level of the centrum semiovale). Cerebral perfusion in each territory and at each level was qualitatively graded as normal (score 1), reduced (score 2 ) or severely reduced (score 3 ) by comparison with contralateral perfusion and normal cerebellar perfusion. ASL and DSC images were assessed separately in random order. For cases of disagreement between the two readers, a consensus reading was appended.

\section{Quantitative image analysis}

For both ASL and DSC datasets, symmetric regions of interest (ROIs) were manually drawn covering the cortex of ACA, MCA and PCA territories (Fig. 2) at the same three levels used for qualitative assessment on each side, as well as regions of interest covering the cerebellar cortex on each side. The rCBF of each territory was defined as the mean ROI signal intensity value of each territory at the three different levels divided by the mean cerebellar signal intensity value. For ASL, quantitative values of CBF within the selected ROIs were noted.

\section{Statistical analyses}


Interreader agreement regarding qualitative cerebral perfusion assessment was evaluated using Cohen's Kappa statistics. A $\kappa$ value greater than 0.81 was interpreted as excellent agreement, values of $0.61-0.80$ were interpreted as good, values of $0.41-0.60$ as moderate, values of $0.21-0.40$ as fair and values less than 0.20 as poor agreement.

Correlation between ASL and DSC was assessed with Spearman's rank correlation for qualitative perfusion scores and with Pearson's correlation coefficient for $\mathrm{rCBF}$, as $\mathrm{rCBF}$ values were distributed normally according to Kolmogorov-Smirnov testing.

For both ASL and DSC independently, rCBF of territories with normal qualitative perfusion versus rCBF of territories with reduced perfusion were compared using student's T tests.

Sensitivity, specificity and accuracy of ASL for the detection of reduced perfusion per territory were calculated from Chi-squared tables of contingency with DSC serving as the standard of reference. 


\section{Results}

\section{Qualitative image analysis}

Diagnostic image quality was achieved for both ASL and DSC acquisitions in all children as evaluated by both readers. Interreader agreement regarding the qualitative visual perfusion assessment was good for both ASL and DSC data (ASL: $\kappa=0.65$; DSC $\kappa=0.62$ ).

Visually reduced qualitative perfusion was found in 19/60 (32\%) territories in 7/10 Patients with ASL (ACA: 7/20, MCA: 10/20, PCA: 2/20) and in 17/60 (28\%) territories in the same 7/10 patients with DSC (ACA: 7/20, MCA: 8/20, PCA: 2/20).

Qualitative perfusion scores of ASL and DSC showed a strong and significant correlation $(\rho=0.77 ; \mathrm{p}<0.001)$.

\section{Quantitative image analysis}

ASL and DSC showed a strong and significant correlation regarding $\mathrm{rCBF}$ values $(\mathrm{r}=0.79$; $\mathrm{p}<0.001$; Fig. 3). Mean rCBF of all territories was not significantly different between ASL and DSC (ASL: $0.93 \pm 0.16$; DSC: $0.92 \pm 0.12 ; \mathrm{p}=0.55$ ).

Territories with normal perfusion scores by qualitative visual assessment showed similar rCBF values in ASL and DSC with no significant differences (ASL: $1.01 \pm 0.08$; DSC: $0.97 \pm$ 0.08; $\mathrm{p}=0.51)$. Furthermore, no significant differences between ASL and DSC were found in territories with reduced perfusion scores by qualitative visual assessment (ASL: $0.77 \pm 0.17$; DSC: $0.80 \pm 0.12 ; \mathrm{p}=0.48)$. 
Mean rCBF was significantly higher in territories with normal perfusion in the qualitative assessment compared to those with reduced perfusion for both ASL and DSC (ASL: 1.01 \pm 0.08 vs. $0.77 \pm 0.17 ; \mathrm{p}<0.001 ; \mathrm{DSC}: 0.97 \pm 0.08$ vs. $0.80 \pm 0.12 ; \mathrm{p}<0.001)$.

Quantitative CBF as calculated from ASL data was significantly higher in territories with normal perfusion compared to those with reduced perfusion in the qualitative assessment

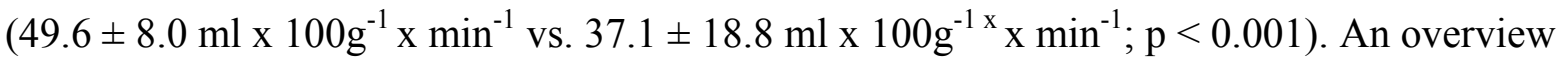
of the results is provided in table 1.

ASL showed a sensitivity of $94 \%$, Specificity of $93 \%$ and accuracy of $93 \%$ for the detection of reduced perfusion per territory with DSC serving as the standard of reference (Fig. 4). 


\section{Discussion}

In children with moyamoya disease, assessment of cerebral perfusion is of interest both for initial evaluation prior to possible measures of revascularization as well as for postoperative follow-up. Nuclear medicine techniques such as SPECT or PET have been shown to be valuable in the assessment of cerebral perfusion and follow-up of patients with moyamoya disease, correlating with the clinical course of the condition [18-22]. However, these methods are associated with a limited availability and considerable radiation doses, which are of concern especially in a pediatric population potentially undergoing serial examinations. The more readily available option of CT perfusion imaging [23] is similarly limited in its use in children due to the radiation burden associated with the method. As a modality not associated with ionizing radiation, MRI has emerged as a valuable method for cerebral perfusion imaging in the assessment of children with moyamoya disease.

To date, DSC remains the most widely applied technique for cerebral MRI perfusion imaging in patients with moyamoya disease. Regarding its use in preoperative assessment, Calamante et al. showed that DSC revealed alterations in cerebral perfusion even in structurally normal appearing cerebral parenchyma in patients with moyamoya disease and suggested that the delineation of areas of abnormal perfusion, in combination with clinical symptomatology could direct the target for measures of revascularization [24]. For postoperative follow-up of patients with moyamoya disease, a study including thirteen children and young adults found a significant reduction of time to peak calculated from DSC 
data in MCA territories following revascularization by EDAS. As time to peak in the basal ganglia showed no significant change, the changes in the MCA territory were interpreted as a marker for the development of collateral circulation from the external carotid artery via the EDAS [25]. In a large study including 67 children with moyamoya disease, it could further be demonstrated that time to peak as well as relative cerebral blood volume significantly decreased after revascularization surgery which was attributed to quicker parenchymal perfusion after revascularization and a decrease of collateral vessel formation. Additionally, time to peak in DSC imaging was significantly different between clinical outcome categories [9].

Recently, the use of ASL for the assessment of patients with moyamoya disease has been reported in various studies of different applications. Noguchi et al. found that CBF as calculated from ASL data in patients with moyamoya disease showed a significant correlation with CBF derived from 123-N-isopropyl-p-iodoamphetamine ( ${ }^{123} \mathrm{I}$-IMP) SPECT, although absolute values of CBF were slightly lower in ASL. This was attributed to delayed arterial transit time caused by steno-occlusive changes and dispersion through collateral vessels [10]. Sugino et al. investigated the use of ASL for the evaluation of focal cerebral hyperperfusion which can occur during the acute stage after STA-MCA bypass surgery - potentially causing transient neurologic deterioration. They found a strong correlation of the asymmetry ratio of rCBF between ASL and ${ }^{123}$ I-IMP SPECT [26]. As a further application, Saida et al. showed that selective ASL with a labeling slab positioned at the external carotid artery was able to demonstrate blood flow to cerebral parenchyma postoperatively via the STA-MCA bypass graft [27]. Some of these studies show potential applications of ASL beyond the possibilities 
of DSC, highlighting the need to assess the comparability of ASL and the more widely accepted and applied DSC.

However, to the best of our knowledge, there is no study in which the correlation of rCBF between ASL and the more commonly applied DSC in children with moyamoya disease has been investigated. In order to compare rCBF values between ASL and DSC data we chose to report the values as the ratio of cortical signal intensity of a given supratentorial vascular territory to the mean signal intensity of the bilateral cerebellar cortex. Unlike using supratentorial left/right rCBF ratios, this approach compares blood flow of each supratentorial territory to the mean cerebellar blood flow independent of laterality, allowing for a simultaneous assessment of differences of left to right and reduced perfusion of both supratentorial sides compared to mean cerebellar perfusion.

We found that in children with moyamoya disease, rCBF measurements with ASL and DSC showed a strong and highly significant correlation $(r=0.79, p<0.001)$, with ASL providing a high accuracy in the detection of reduced perfusion per territory using DSC as the standard of reference. ASL therefore bears the potential of replacing DSC under certain circumstances, i.e. if the use of contrast agent needs to be avoided. The high correlation between ASL and DSC we found is in concordance with a report in the literature showing correlation coefficients between ASL and DSC of 0.62-0.79 in patients with acute ischemic stroke [28]. The values of quantitative CBF we found were significantly higher in territories with qualitatively normal perfusion, compared to those with reduced perfusion $(49.6 \pm 8.0 \mathrm{ml}$ x $100 \mathrm{~g}^{-1} \times \min ^{-1}$ vs. $\left.37.1 \pm 18.8 \mathrm{ml} \times 100 \mathrm{~g}^{-1} \times \min ^{-1} ; \mathrm{p}<0.001\right)$. However, it must be noted that we did not have a standard of reference such as SPECT, PET or perfusion CT with which to validate our quantitative results. The CBF values we found in territories with normal- 
appearing perfusion are in concordance with normal ASL gray-matter CBF values in adults $\left(58 \pm 4 \mathrm{ml} \times 100 \mathrm{~g}^{-1} \times \min ^{-1}\right)$, although lower than would be expected in children [29]. Rather than indicating globally reduced perfusion (as the cerebellum, which is generally spared in moyamoya disease showed similar values) this finding is most probably caused by partial inclusion of peripheral white matter in the ROIs drawn over the cortex of each territory at a specific level. Rather than using multiple smaller ROIs exclusively positioned in the cortex, we chose to use large symmetric ROIs to minimize the possible bias of small sample sizes in territories with potentially inhomogeneous perfusion.

An important limitation of both ASL and DSC is that results are influenced by the presence of stenoocclusive disease and the presence of collateral moyamoya vessels as these may lead to a delay in arrival times of the labeled arterial water spins or the gadolinium bolus, respectively. For DSC it has been shown that the effect of bolus arrival delay and bolus dispersion due to the presence of collateral vessels may lead to underestimation of rCBF and the overestimation of mean transit time (MTT) as calculated by deconvolution [30]. For ASL imaging, long transit times caused by the presence of collateral vessels may lead to a loss of perfusion signal, if the labeled blood does not reach the imaging slices before the start of image acquisition. This effect results in artificially low CBF values [31]. Alternatively, if arterial transit times are increased such that spins remain in the vasculature at the time of image readout, bright intraluminal signal can give the appearance of increased perfusion due to the longer $\mathrm{T} 1$ of blood relative to tissue, since the label decays more slowly if the spins remain intravascular [32]. The visibility of this artifact will vary according to the ASL acquisition method, and whether flow-crushing gradients are implemented to suppress signal from intravascular spins before imaging. The presence of intraluminal signal is also typically 
associated with an underestimate of perfusion in the distal field, but in the present study, intraluminal artefacts were not observed in the vicinity of the ROIs.

Cerebral blood flow may therefore be underestimated by the effects of arrival delay in both techniques, however quantification of CBF in DSC is additionally complicated by other factors, including uncertainties in the arterial input function (i.e. due to partial volume effects, flow artifacts and inflow effects) and the non-linear relationship between contrast concentration and $\mathrm{T} 2 / \mathrm{T} 2 *$ relaxivity $[12 ; 13]$. The arterial input function is required for absolute quantification of CBF in DSC, which was not attempted in the present study. The combined use of ASL and DSC may improve quantitative CBF measurements, as shown in a study by Zaharchuk et al. introducing a calibration method using CBF from ASL in regions with short transit times to provide a patient-specific conversion factor for the quantification of rCBF from DSC [33].

Although CBF values in ASL may be underestimated by arrival delay due to stenoocclusive disease and formation of collaterals, the lack of dependence from contrastmaterial related issues can be expected to render serial follow-up examinations more comparable in children with moyamoya disease. A further benefit of ASL is its repeatability, which could be used for combined acetazolamide-challenge / rest examinations to define the cerebral blood flow reserve, an important parameter for both preoperative assessment and monitoring disease progression. Such examinations have been performed with SPECT, PET and Xenon-enhanced CT for almost 20 years $[34 ; 35]$ and have been shown to predict clinical outcome postoperatively [19]. A non-invasive, non-ionizing modality for the assessment of cerebral perfusion reserve would be more than desirable - especially for the pediatric 
population - and further studies are warranted to investigate whether ASL can provide such information.

\section{Limitations}

We acknowledge the following limitations of our study: Firstly, our patient population is too small to allow for sub-analysis of findings in pre- and postoperative patients. Secondly, we did not have serial examinations available to assess the use of ASL in follow-up imaging. Thirdly, we did not have a standard of reference such as PET, SPECT or perfusion-CT available to validate the ASL results of quantitative CBF. Fourthly, an inherent limitation of ASL is that it does not allow for the calculation of mean transit time and cerebral blood volume, therefore these parameters could not be compared between ASL and DSC. And finally, in this retrospective study we could not assess the use of azetazolamide-challenge/rest ASL, which appears to be an interesting possible application of ASL, warranting further studies.

\section{Conclusion}

Our results demonstrate that the assessment of relative cerebral blood flow with ASL in children with moyamoya disease shows a strong and significant correlation with the currently more widely used method of DSC both qualitatively and quantitatively. ASL allows for the detection of reduced perfusion per vascular territory with good accuracy using DSC as the standard of reference and additionally offers the possibility of quantitative cerebral blood flow assessment. 


\section{Conflict of interest}

We declare that we have no conflict of interest. 


\section{References}

1 Suzuki J, Takaku A (1969) Cerebrovascular "moyamoya" disease. Disease showing abnormal net-like vessels in base of brain. Arch Neurol, 20(3):288-299

2 Suzuki J, Kodama N (1983) Moyamoya disease--a review. Stroke, 14(1):104-109

3 Fujita K, Tamaki N, Matsumoto S (1986) Surgical treatment of moyamoya disease in children: which is more effective procedure, EDAS or EMS? Childs Nerv Syst, 2(3):134-138

4 Matsushima T, Inoue T, Suzuki SO, Fujii K, Fukui M, Hasuo K (1992) Surgical treatment of moyamoya disease in pediatric patients--comparison between the results of indirect and direct revascularization procedures. Neurosurgery, 31(3):401-405

5 Patel NN, Mangano FT, Klimo P, Jr. (2010) Indirect revascularization techniques for treating moyamoya disease. Neurosurg Clin N Am, 21(3):553-563

6 Khan N, Schuknecht B, Boltshauser E, et al. (2003) Moyamoya disease and Moyamoya syndrome: experience in Europe; choice of revascularisation procedures. Acta Neurochir (Wien), 145(12):1061-1071; discussion 1071

7 Huisman TA, Sorensen AG (2004) Perfusion-weighted magnetic resonance imaging of the brain: techniques and application in children. Eur Radiol, 14(1):59-72

8 Tzika AA, Robertson RL, Barnes PD, et al. (1997) Childhood moyamoya disease: hemodynamic MRI. Pediatr Radiol, 27(9):727-735

9 Yun TJ, Cheon JE, Na DG, et al. (2009) Childhood moyamoya disease: quantitative evaluation of perfusion MR imaging--correlation with clinical outcome after revascularization surgery. Radiology, 251(1):216-223

10 Noguchi T, Kawashima M, Irie H, et al. (2011) Arterial spin-labeling MR imaging in moyamoya disease compared with SPECT imaging. Eur J Radiol, 80(3):e557-562 
11 Bokkers RP, Bremmer JP, van Berckel BN, et al. (2010) Arterial spin labeling perfusion MRI at multiple delay times: a correlative study with $\mathrm{H}(2)(15) \mathrm{O}$ positron emission tomography in patients with symptomatic carotid artery occlusion. J Cereb Blood Flow Metab, 30(1):222-229

12 Jochimsen TH, Newbould RD, Skare ST, et al. (2007) Identifying systematic errors in quantitative dynamic-susceptibility contrast perfusion imaging by high-resolution multi-echo parallel EPI. NMR Biomed, 20(4):429-438

13 van Osch MJ, van der Grond J, Bakker CJ (2005) Partial volume effects on arterial input functions: shape and amplitude distortions and their correction. J Magn Reson Imaging, 22(6):704-709

14 Dai W, Garcia D, de Bazelaire C, Alsop DC (2008) Continuous flow-driven inversion for arterial spin labeling using pulsed radio frequency and gradient fields. Magn Reson Med, 60(6):1488-1497

15 Alsop DC, Detre JA (1996) Reduced transit-time sensitivity in noninvasive magnetic resonance imaging of human cerebral blood flow. J Cereb Blood Flow Metab, $16(6): 1236-1249$

16 Wang J, Zhang Y, Wolf RL, Roc AC, Alsop DC, Detre JA (2005) Amplitudemodulated continuous arterial spin-labeling 3.0-T perfusion MR imaging with a single coil: feasibility study. Radiology, 235(1):218-228

17 Jarnum H, Steffensen EG, Knutsson L, et al. (2010) Perfusion MRI of brain tumours: a comparative study of pseudo-continuous arterial spin labelling and dynamic susceptibility contrast imaging. Neuroradiology, 52(4):307-317

18 Feole JB, Ali A, Fordham EW, Huckman M, Shenker DM (1993) Serial SPECT imaging in moyamoya using I-123 IMP. A method of noninvasive evaluation and follow-up. Clin Nucl Med, 18(1):43-45 
19 So Y, Lee HY, Kim SK, et al. (2005) Prediction of the clinical outcome of pediatric moyamoya disease with postoperative basal/acetazolamide stress brain perfusion SPECT after revascularization surgery. Stroke, 36(7):1485-1489

20 Kuwabara Y, Ichiya Y, Sasaki M, et al. (1997) Cerebral hemodynamics and metabolism in moyamoya disease--a positron emission tomography study. Clin Neurol Neurosurg, 99 Suppl 2:S74-78

21 Weber B, Westera G, Treyer V, Burger C, Khan N, Buck A (2004) Constant-infusion $\mathrm{H}(2) 15 \mathrm{O}$ PET and acetazolamide challenge in the assessment of cerebral perfusion status. J Nucl Med, 45(8):1344-1350

22 Song YS, Oh SW, Kim YK, Kim SK, Wang KC, Lee DS (2012) Hemodynamic improvement of anterior cerebral artery territory perfusion induced by bifrontal encephalo(periosteal) synangiosis in pediatric patients with moyamoya disease: a study with brain perfusion SPECT. Ann Nucl Med, 26(1):47-57

23 Kang KH, Kim HS, Kim SY (2008) Quantitative cerebrovascular reserve measured by acetazolamide-challenged dynamic CT perfusion in ischemic adult Moyamoya disease: initial experience with angiographic correlation. AJNR Am J Neuroradiol, 29(8):1487-1493

24 Calamante F, Ganesan V, Kirkham FJ, et al. (2001) MR perfusion imaging in Moyamoya Syndrome: potential implications for clinical evaluation of occlusive cerebrovascular disease. Stroke, 32(12):2810-2816

25 Lee SK, Kim DI, Jeong EK, et al. (2003) Postoperative evaluation of moyamoya disease with perfusion-weighted MR imaging: initial experience. AJNR Am J Neuroradiol, 24(4):741-747

26 Sugino T, Mikami T, Miyata K, Suzuki K, Houkin K, Mikuni N (2012) Arterial SpinLabeling Magnetic Resonance Imaging after Revascularization of Moyamoya Disease. J Stroke Cerebrovasc Dis 
27 Saida T, Masumoto T, Nakai Y, Shiigai M, Matsumura A, Minami M (2012)

Moyamoya disease: evaluation of postoperative revascularization using multiphase selective arterial spin labeling MRI. J Comput Assist Tomogr, 36(1):143-149

28 Wang DJ, Alger JR, Qiao JX, et al. (2012) The value of arterial spin-labeled perfusion imaging in acute ischemic stroke: comparison with dynamic susceptibility contrastenhanced MRI. Stroke, 43(4):1018-1024

29 Biagi L, Abbruzzese A, Bianchi MC, Alsop DC, Del Guerra A, Tosetti M (2007) Age dependence of cerebral perfusion assessed by magnetic resonance continuous arterial spin labeling. J Magn Reson Imaging, 25(4):696-702

30 Calamante F, Gadian DG, Connelly A (2000) Delay and dispersion effects in dynamic susceptibility contrast MRI: simulations using singular value decomposition. Magn Reson Med, 44(3):466-473

31 Calamante F, Thomas DL, Pell GS, Wiersma J, Turner R (1999) Measuring cerebral blood flow using magnetic resonance imaging techniques. J Cereb Blood Flow Metab, 19(7):701-735

32 Detre JA, Alsop DC, Vives LR, Maccotta L, Teener JW, Raps EC (1998) Noninvasive MRI evaluation of cerebral blood flow in cerebrovascular disease. Neurology, 50(3):633-641

33 Zaharchuk G, Straka M, Marks MP, Albers GW, Moseley ME, Bammer R (2010) Combined arterial spin label and dynamic susceptibility contrast measurement of cerebral blood flow. Magn Reson Med, 63(6):1548-1556

34 Hoshi H, Ohnishi T, Jinnouchi S, et al. (1994) Cerebral blood flow study in patients with moyamoya disease evaluated by IMP SPECT. J Nucl Med, 35(1):44-50

35 Nariai T, Suzuki R, Hirakawa K, Maehara T, Ishii K, Senda M (1995) Vascular reserve in chronic cerebral ischemia measured by the acetazolamide challenge test: 
comparison with positron emission tomography. AJNR Am J Neuroradiol, 16(3):563570 


\section{Tables and Figure legends}

Table 1: Overview of results of quantitative perfusion analysis

ASL

DSC p

\begin{tabular}{|c|c|c|c|c|c|c|}
\hline $\begin{array}{l}\text { All } \\
\text { territories }\end{array}$ & $\mathrm{rCBF}$ & $0.93 \pm 0.16$ & $(n=60)$ & $0.92 \pm 0.12$ & $(n=60)$ & 0.55 \\
\hline & $\mathrm{rCBF}$ & $1.01 \pm 0.08$ & $(n=41)$ & $0.97 \pm 0.08$ & $(n=43)$ & 0.51 \\
\hline $\begin{array}{l}\text { Territories } \\
\text { with } \\
\text { normal } \\
\text { perfusion* }\end{array}$ & $\begin{array}{l}\mathrm{CBF} \\
\left(\mathrm{ml} \times 100 \mathrm{~g}^{-1} \times \min ^{-1}\right)\end{array}$ & $49.6 \pm 8.0$ & & $\mathrm{n} / \mathrm{a}$ & & \\
\hline & $\mathrm{rCBF}$ & $0.77 \pm 0.17$ & $(n=19)$ & $0.80 \pm 0.12$ & $(n=17)$ & 0.48 \\
\hline $\begin{array}{l}\text { Territories } \\
\text { with } \\
\text { reduced } \\
\text { perfusion* }\end{array}$ & $\begin{array}{l}\mathrm{CBF} \\
\left(\mathrm{ml} \times 100 \mathrm{~g}^{-1} \times \mathrm{min}^{-1}\right)\end{array}$ & $37.1 \pm 18.8$ & & $\mathrm{n} / \mathrm{a}$ & & \\
\hline
\end{tabular}

ASL: arterial spin labeling; DSC: dynamic susceptibility contrast imaging; rCBF: relative cerebral blood flow calculated as ratio to cerebellar perfusion; CBF: absolute cerebral blood flow as calculated from ASL data.

* Between territories with normal and reduced perfusion as assessed by qualitative visual scoring, differences in $\mathrm{rCBF}$ were significant for both ASL and DSC (each $\mathrm{p}<0.001$ ). 
Fig. 1: 3D-volume rendered reconstruction of time-of-flight MR-angiography of the anterior circulation in a 5-year-old girl with moyamoya disease. Note the extensive collateral moyamoya vessels on the left (white arrowheads). On the right there is a high-grade stenosis of the supraclinoid internal cerebral artery and proximal middle cerebral artery (white arrow) without appreciable moyamoya vessels.

Fig. 2: Imaging example of region of interest (ROI) placement for $\mathrm{rCBF}$ measurements in the source image (left) and corresponding rCBF map (right) at the level of the thalami in a 10year-old patient with moyamoya disease.

Fig. 3: Correlation of rCBF between ASL and DSC

Fig. 4: Images of T2-weighted, diffusion-weighted, ASL and DSC sequences at the levels of the tentorium, thalami and centrum semiovale in the same 5-year-old girl with moyamoya disease depicted in figure 1. T2- and diffusion-weighted images show no signs of chronic or acute ischemia, however both ASL and DSC demonstrate inhomogenously reduced perfusion in the anterior cerebral artery territories bilaterally as well as the right middle cerebral artery territory, presumably caused by the high-grade supraclinoid stenosis on the right without adequate collateralization (see figure 1).Most probably due to local sub-territory perfusion inhomogeneities, the reduced perfusion in the left ACA territory appears more pronounced on the left on the lowest slice and more pronounced on the right on the upper two slices in the 
DSC images by lateral comparison, while overall being reduced on both sides. Furthermore, although the differences between MCA territories appear more pronounced at the level of the centrum semiovale in DSC compared to ASL, the reduced perfusion on the right can be appreciated in both modalities. 